\title{
Évaluation pluri-critères de l'agriculture biologique en Afrique subsaharienne par élicitation probabiliste des connaissances d'experts
}

\author{
Landy Andriamampianina ${ }^{1, *}$, Ludovic Temple ${ }^{2,4}$, Hubert de Bon $^{3,4}$, Eric Malézieux ${ }^{3,4}$ et David Makowski ${ }^{5}$ \\ 1 Toulouse School of Economics, 21 Allée de Brienne, 31000 Toulouse, France \\ 2 CIRAD, UMR INNOVATION, F-34398 Montpellier, France \\ ${ }^{3}$ CIRAD, UPR HORTSYS, F-34398 Montpellier, France \\ ${ }^{4}$ Univ Montpellier, Montpellier, France \\ ${ }^{5}$ INRA AgroParisTech, Université de Paris-Saclay, UMR Agronomie, F-78850 Thiverval-Grignon, France
}

\begin{abstract}
Résumé - De nombreux agriculteurs africains pratiquent des formes d'agriculture potentiellement qualifiables de «biologiques ». Pourtant, la capacité de l'agriculture biologique à répondre aux enjeux de la sécurité alimentaire en Afrique est encore mal connue, car il existe peu de références expérimentales disponibles dans cette région. L'élicitation probabiliste est une méthode permettant de rendre compte de manière précise des connaissances d'experts sur une ou plusieurs quantités d'intérêt, et de décrire les niveaux d'incertitude associés. L'objectif de cette étude est de montrer comment cette approche permet de pallier le manque de données expérimentales quantitatives sur l'agriculture biologique en Afrique. L'élicitation probabiliste a été appliquée au Sénégal, au Burkina Faso et au Cameroun, en mobilisant 17 experts pour comparer les performances des agricultures biologique et conventionnelle sur la sécurité alimentaire locale en Afrique subsaharienne, en considérant plusieurs indicateurs quantitatifs. Les résultats montrent que les rendements des systèmes biologiques sont inférieurs d'environ $41 \%$ à ceux des systèmes conventionnels, tandis que les prix des produits issus de l'agriculture biologique sont supérieurs de $34 \%$ à ceux des produits issus de l'agriculture conventionnelle. Nos résultats montrent également que le coût en main-d'œuvre, mesuré en nombre d'homme-jours par unité de surface cultivée, est plus élevé dans les systèmes biologiques. Cette étude est la première à montrer l'intérêt de l'élicitation probabiliste des connaissances d'experts pour évaluer des systèmes de culture dans des situations où les données expérimentales sont rares ou peu fiables.
\end{abstract}

Mots clés : agriculture biologique / sécurité alimentaire / innovation / méthode statistique / Afrique subsaharienne

\begin{abstract}
Multi-criteria evaluation of organic agriculture in sub-Saharan Africa using probabilistic elicitation of expert knowledge. Many African agricultural systems can potentially be described as "organic". However, the capacity of organic agriculture to address food security issues in Africa is poorly known, as only few experimental studies are available in this region. Probabilistic elicitation is used here to represent knowledge of experts on one or more quantities of interest in a quantitative manner, and to describe the associated levels of uncertainty. The objective of this study is to explore how probabilistic elicitation of expert knowledge could be used to overcome the lack of quantitative experimental data on the performance of organic agriculture in Africa. This approach was applied in Senegal, Burkina Faso and Cameroon where 17 experts were interviewed on the relative performance of organic and conventional agriculture on different aspects of the local food security in sub-Saharan Africa. Our results show that the yields of organic systems are about $41 \%$ lower than the yields of conventional systems, while the prices of organic products are $34 \%$ higher than prices of products from conventional agriculture. According to the experts, the cost of labor, measured in man-days per unit of cultivated land area, is higher in organic systems. This study is the first to demonstrate the interest of the probabilistic elicitation of expert knowledge to evaluate cropping systems in situations where the experimental data are scarce or unreliable.
\end{abstract}

Keywords: organic farming / food security / innovation / statistical method / sub-Saharan Africa

*Auteur correspondant : andria.landy@gmail.com 


\section{Introduction}

L'Afrique subsaharienne est caractérisée par la plus forte prévalence d'insécurité alimentaire dans le monde alors que l'agriculture est la principale activité économique de cette région. Bien que l'agriculture d'Afrique subsaharienne utilise globalement peu d'intrants chimiques comparativement aux agricultures des pays industriels et émergents, la consommation de pesticides peut devenir dangereuse pour les écosystèmes, mais aussi et surtout pour la santé humaine dans certaines conditions, en particulier dans le domaine horticole (de Bon et al., 2014). Par ailleurs, au regard des externalités négatives de l'agriculture industrielle - pollution, changement climatique, crises sanitaires (Temple et al., 2015) -, le marché mondial des produits issus de l'agriculture biologique est en pleine expansion. Bien que non-certifiés, beaucoup d'agriculteurs africains pratiquent des formes d'agricultures qui peuvent être potentiellement qualifiées de « biologiques », car ils n'utilisent pas ou très peu d'intrants chimiques.

La capacité de l'agriculture biologique à répondre aux enjeux que pose la sécurité alimentaire (Touzard et Temple, 2012) en Afrique reste mal connue. La faiblesse des rendements de l'agriculture biologique par rapport à l'agriculture conventionnelle est fréquemment soulignée (Berezow, 2017), mais peu de références expérimentales fiables sont disponibles en Afrique (Lesur-Dumoulin et al., 2017 ; Seufert et Ramankutty, 2017). La mise en place de nouveaux dispositifs expérimentaux pour explorer les potentialités agronomiques et économiques des productions biologiques africaines est limitée par les faibles investissements des pays africains dans la recherche agronomique, dus notamment à de fortes instabilités macro-économiques. Les études quantitatives sur les performances de l'agriculture biologique sont encore très rares en Afrique.

L'objectif de cette étude est de montrer que l'élicitation probabiliste des connaissances d'experts peut être utilisée pour pallier le manque de données expérimentales pour analyser les relations entre l'agriculture biologique et la sécurité alimentaire. Cette méthode permet d'exprimer l'opinion subjective des experts sous forme quantitative et de représenter l'incertitude associée aux connaissances disponibles (Garthwaite et al., 2005). Elle est de plus en plus utilisée dans le cadre d'expertises scientifiques par les agences d'évaluation des risques sanitaires (EFSA, 2014; Makowski et al., 2016), mais encore très rarement dans les instituts agronomiques de recherche. Nous proposons ici de l'utiliser pour comparer la contribution de l'agriculture biologique et de l'agriculture conventionnelle à la sécurité alimentaire locale en Afrique subsaharienne.

\section{2 Élicitation probabiliste pour analyser les performances de l'agriculture biologique en Afrique}

\subsection{Objectif de l'élicitation}

«L'élicitation est un processus visant à formaliser la connaissance et les croyances d'une personne au sujet d'une ou plusieurs quantités incertaines sous la forme d'une distribution de probabilités de cette/ces quantité(s)» (Garthwaite et al., 2005). La distribution de probabilités obtenue par élicitation peut être utilisée seule, ou en combinaison avec des données dans le cadre de la statistique bayésienne (Albert et al., 2015).

Un expert est une personne ayant acquis une bonne compétence dans une profession ou une discipline, grâce à une longue expérience lui permettant de faire des jugements pertinents sur un sujet (Albert et al., 2015). Cependant, bien souvent, l'expert ne connaît pas parfaitement la valeur réelle de la quantité considérée. Dans ce cas, son incertitude peut être représentée par une distribution de probabilités au lieu d'une valeur unique (Morris et al., 2014). Cette distribution permet de calculer la probabilité qu'une quantité se situe dans une fourchette de valeurs donnée (Cohen et Chechile, 1997). En d'autres termes, elle représente les niveaux de confiance d'un expert dans les valeurs que prendront la quantité étudiée (O’Hagan, 2012), ou encore la gamme de variabilité estimée par cet expert pour la quantité considérée. D'un point de vue opérationnel, l'élicitation est réalisée lors d'un entretien avec l'expert dont les modalités sont décrites ci-dessous.

\subsection{Définition des quantités élicitées et du contexte}

La sécurité alimentaire est généralement définie autour de 4 piliers : disponibilité, accessibilité, utilisation et stabilité (Dury et al., 2017). Nous avons sélectionné des indicateurs quantitatifs nous permettant d'évaluer respectivement les performances des agricultures biologique et conventionnelle sur les trois premiers piliers. Nous n'avons pas considéré d'indicateur pour le pilier «stabilité ».

Pour le pilier «disponibilité », nous utilisons l'indicateur «rendement» exprimé en tonnes par hectare. Pour le pilier «accessibilité », nous utilisons trois indicateurs :

- le coût des intrants, défini comme le coût total des intrants utilisés dans la production (semences, engrais, produits phytosanitaires, à l'exclusion du travail), mesuré en unité monétaire (francs CFA);

- le coût du travail, mesuré en nombre d'homme-jours par unité de surface cultivée;

- le prix bord champ des produits agricoles (francs CFA).

Nous avons retenu quatre indicateurs liés au pilier «utilisation», correspondant soit à la qualité sanitaire des aliments, soit à leur qualité nutritionnelle. Pour la qualité sanitaire, les indicateurs sont:

- la durée de conservation de la culture étudiée, c'est-à-dire la durée durant laquelle un produit alimentaire peut être stocké sans devenir impropre à la consommation;

- le risque (en pourcentage) de contamination de la culture étudiée par les mycotoxines.

Pour la qualité nutritionnelle, nous avons choisi des indicateurs de sécurité alimentaire:

- le Score de diversité alimentaire des ménages (SDAM), qui mesure la capacité d'un ménage à avoir accès à une grande variété d'aliments. Le SDAM représente le nombre de groupes d'aliments qu'un ménage a consommé au cours 


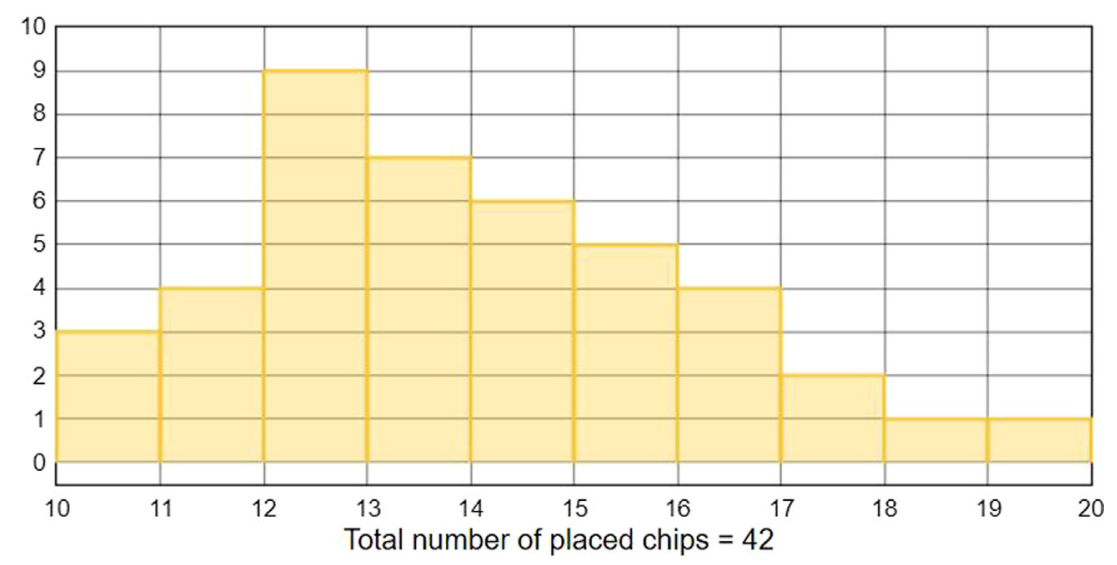

Fig. 1. Élicitation d'une distribution de probabilités à l'aide de la méthode de la roulette. L'axe des ordonnées présente le nombre de jetons $(0$ à 10) et l'axe des abscisses présente les valeurs possibles de l'indicateur. Les jetons sont représentés par les cubes en orange. Il faut cliquer sur chaque case de la grille pour les faire apparaître. Sur l'image, nous pouvons voir que l'expert pense qu'il existe des chances très élevées que l'indicateur se situe entre 12 et 13 et des chances très faibles qu'il soit entre 19 et 20 .

Fig. 1. Eliciting a probability distribution using the roulette method.

des 24 heures précédentes. Plus le score est élevé, plus le régime alimentaire du ménage est diversifié;

- la fréquence de consommation d'aliments riches en vitamine A et en fer (mesurée en nombre de jours par semaine).

Ces indicateurs correspondent à des quantités qui sont mal connues, notamment en Afrique, mais pour lesquels des experts régionaux disposent de connaissances, même si elles sont incertaines.

L'élicitation nécessite de définir un contexte formel pour aider l'expert à mobiliser ses connaissances sur un indicateur donné. Dans notre cas, le contexte est défini par trois éléments : la localisation géographique, les espèces cultivées et des définitions précises des systèmes biologique et conventionnel. Notre étude concerne le Cameroun, le Sénégal et le Burkina Faso, et couvre ainsi une large variabilité climatique en Afrique. Nous avons sélectionné cinq cultures produisant des denrées alimentaires traditionnelles dans ces pays : le sorgho, l'igname, le manioc, l'oignon et la mangue. Dans notre étude, les systèmes biologiques sont définis comme suivant les principes définis par l'International Federation of Organic Agriculture Movements (IFOAM): santé, écologie, équité et préservation pour les générations futures. Les systèmes conventionnels se réfèrent ici à des systèmes s'appuyant sur l'utilisation d'intrants chimiques.

Afin de choisir les experts à interroger, nous avons défini des profils d'expertise détaillant les qualifications requises sur les cultures, les systèmes et les pays. Nous avons essayé de mobiliser le plus d'experts possible pour obtenir une couverture représentative de l'éventail des opinions (Morgan, 2014), mais nous étions limités par le nombre d'experts disponibles pendant la période de l'étude. Nous avons demandé aux experts de justifier leurs distributions de probabilités en nous fournissant des éléments factuels.

\section{Démarche et outils utilisés}

Deux outils ont été utilisés pour mener les enquêtes auprès des experts : le logiciel Match Uncertainty Elicitation Tool et un questionnaire.

\subsection{Match Uncertainty Elicitation Tool}

Nous avons réalisé les élicitations à l'aide du logiciel Match Uncertainty Elicitation Tool, produit par Morris et al. (2014). Cet outil est gratuit et disponible en ligne (http://optics. eee.nottingham.ac.uk/match/uncertainty.php).

L'outil propose cinq méthodes d'élicitation et nous avons choisi d'utiliser ici la «méthode de la roulette» (Morris et al., 2014; Oakley, 2010). Cette méthode fournit une représentation très intuitive des connaissances des experts sous la forme d'un histogramme, puis permet d'ajuster une loi de probabilités continue. Elle est appliquée en quatre étapes:

Étape 1. La première étape consiste à demander à l'expert de choisir un indicateur parmi la liste proposée, puis de définir son unité de mesure.

Lors des trois étapes suivantes, l'expert définit une distribution de probabilités pour l'indicateur choisi, séparément pour chaque système. Ces étapes sont donc réalisées deux fois (une fois pour le système conventionnel, une fois pour le système biologique).

Étape 2. On demande à l'expert de fixer les bornes inférieure et supérieure des valeurs de l'indicateur sélectionné. Ces bornes définissent la gamme possible de variation de l'indicateur. L'enquêteur divise cet intervalle en $\mathrm{K}$ sousintervalles de valeurs d'indicateur (axe des $\mathrm{x}$ ). Chaque sousintervalle définit une colonne qui est divisée elle-même en $\mathrm{P}$ cases (axe des y), définissant ainsi une grille comportant un total de K*P cases (Fig. 1). 

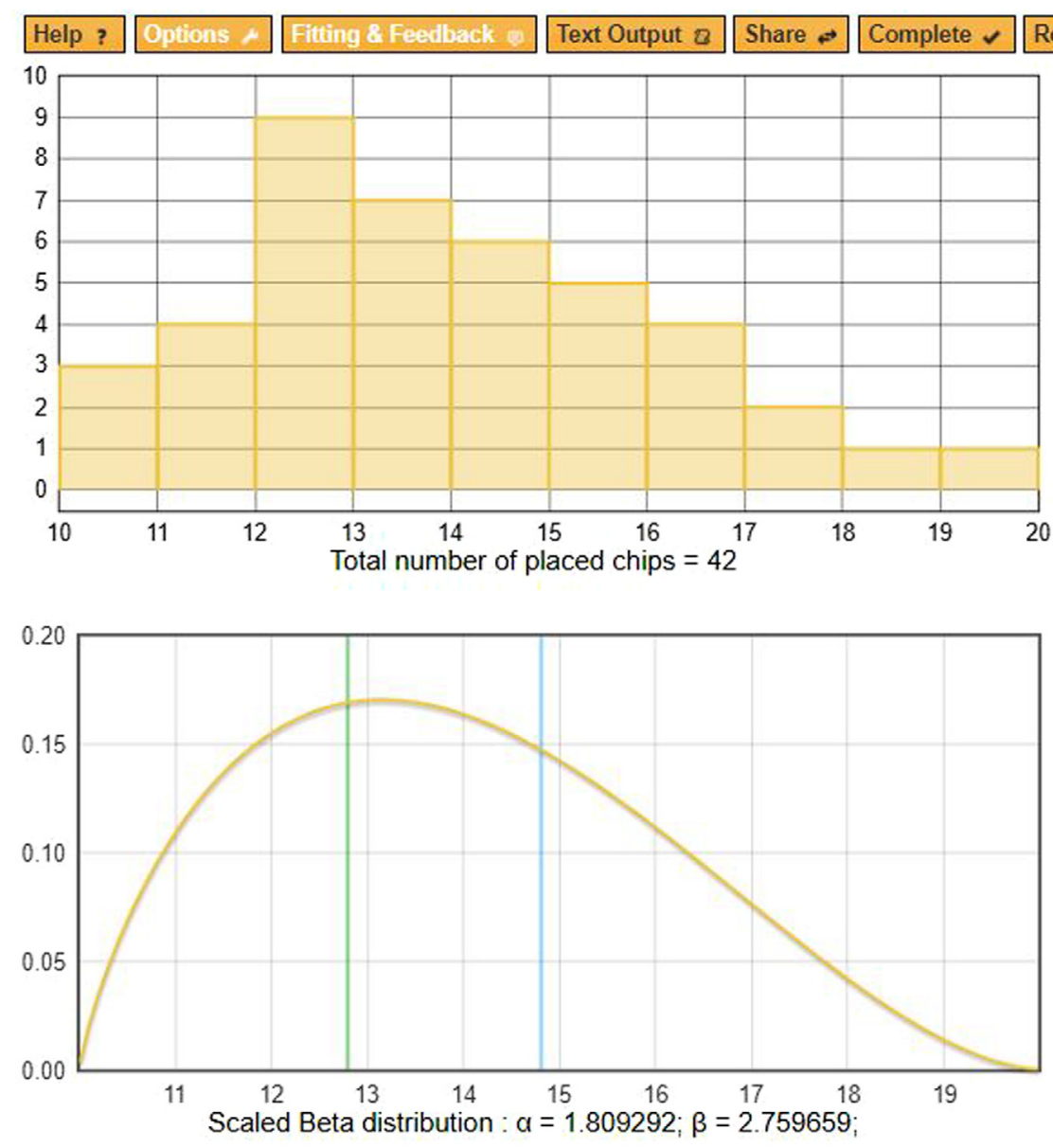

Restart $\$$ About Us $\mathbf{0}$

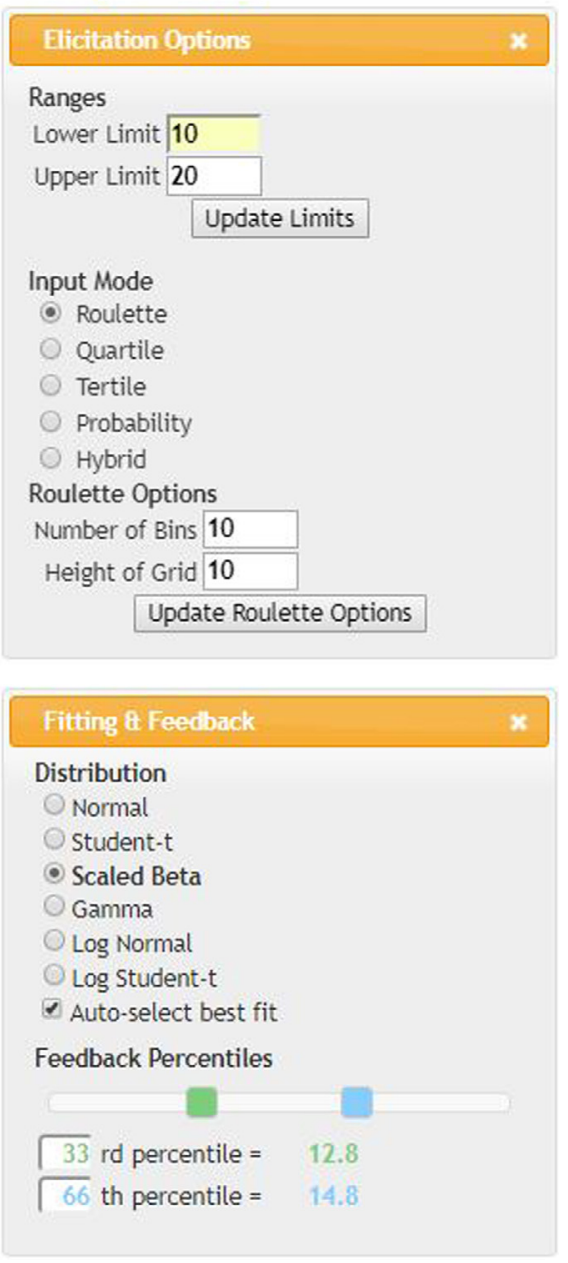

Fig. 2. Ajustement d'une distribution de probabilités à l'histogramme élicité.

Fig. 2. Fitting and feedback.

Étape 3. On demande à l'expert de placer des jetons sur la grille. Le nombre de jetons attribués dans chaque sousintervalle doit refléter la probabilité que la valeur de l'indicateur soit située dans ce sous-intervalle, selon l'expert. Le résultat obtenu correspond à un histogramme (Fig. 1).

Étape 4. Une série de lois de probabilités est ajustée à l'histogramme et celle qui s'ajuste le mieux est sélectionnée (Fig. 2). Les deux terciles de la distribution choisie sont présentés à l'expert; ils représentent les valeurs atteintes une fois sur trois et deux fois sur trois. Si ces valeurs ne donnent pas satisfaction à l'expert, celui-ci modifie l'histogramme (étape 3) et réajuste les lois de probabilités (étape 4). Le processus est répété jusqu'à ce que l'expert soit satisfait.

\subsection{Construction de l'indicateur de comparaison des deux systèmes}

$\mathrm{Au}$ total, 17 questionnaires ont été remplis auprès de 17 experts. Pour chaque localisation*culture et chaque indicateur $\mathrm{X}$, nous disposons de deux distributions de probabilités: une distribution pour la quantité $X_{C}$ dans le système conventionnel et une pour la quantité $X_{B}$ dans le système biologique. A partir de ces deux distributions, nous déterminons la distribution conjointe de $\mathrm{X}_{\mathrm{C}}$ et $\mathrm{X}_{\mathrm{B}}$. Pour cela, nous demandons à l'expert d'éliciter la corrélation entre $X_{C}$ et $X_{B}$, puis nous utilisons cette corrélation pour définir la distribution conjointe de $\mathrm{X}_{\mathrm{C}}$ et $\mathrm{X}_{\mathrm{B}}$ à l'aide d'une copule gaussienne (Clemen et Reilly, 1999; Yan, 2007). Des tirages aléatoires sont ensuite réalisés pour estimer la distribution du ratio $\mathrm{X}_{\mathrm{B}} / \mathrm{X}_{\mathrm{C}}$. L'avantage de cette approche est qu'elle permet à l'expert de travailler avec $X_{C}$ et $X_{B}$ successivement, sans avoir besoin d'éliciter ses connaissances sur le ratio $X_{B} / X_{C}$ directement. Les experts ont généralement des connaissances sur les valeurs de $X_{C}$ et $X_{B}$, et préfèrent donc travailler avec ces quantités plutôt qu'avec leur ratio.

Chaque distribution de ratio est décrite par sa médiane et ses seuils de $1^{\text {er }}$ et $9^{\text {e }}$ déciles bornant un intervalle de crédibilité à $80 \%$. Plus cet intervalle de crédibilité est grand, plus le niveau d'incertitude de l'expert sur la valeur de l'indicateur est élevé. Par opposition, plus l'intervalle de crédibilité est petit, plus le niveau d'incertitude de l'expert est faible. 
Organic to conventional yields ratio by crop and by country

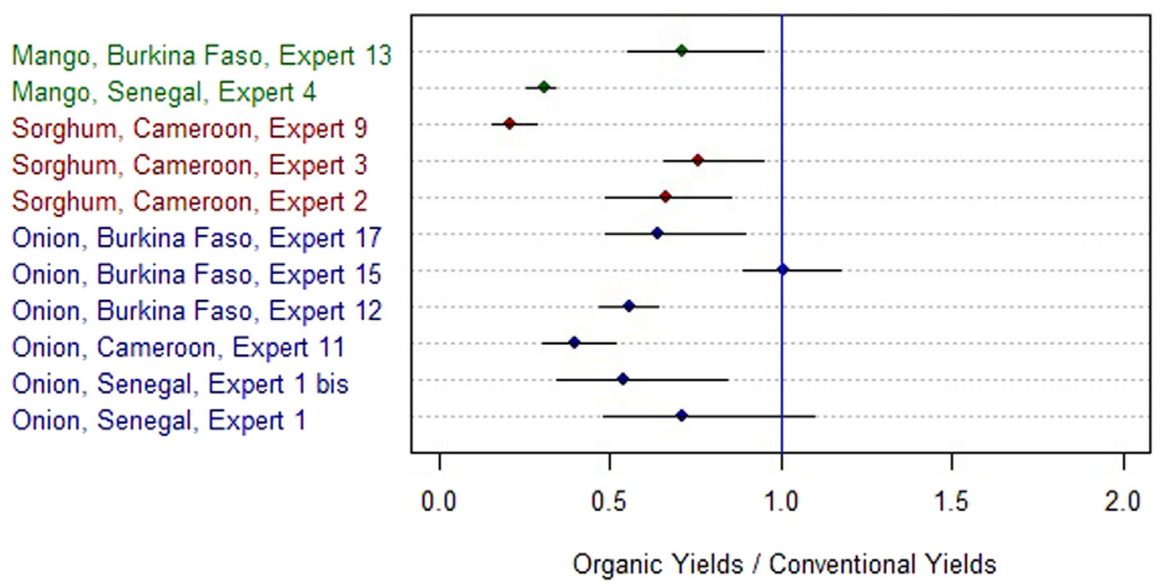

Fig. 3. Médiane et intervalles de crédibilité $(80 \%)$ du ratio biologique/conventionnel des rendements. La ligne bleue représente le seuil pour lequel les rendements biologiques et conventionnels sont identiques. Chaque point indique la médiane de la distribution élicitée.

Fig. 3. Median and credibility intervals (80\%) of organic to conventional yields ratio.

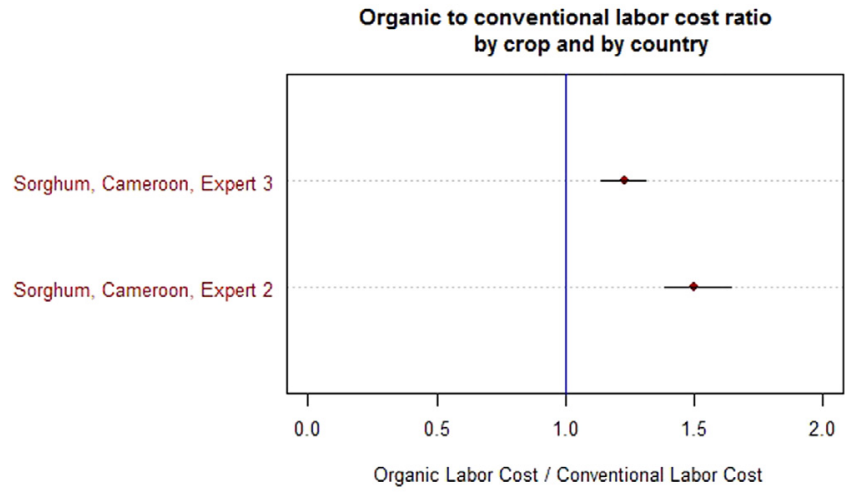

Fig. 4. Médiane et intervalles de crédibilité $(80 \%)$ du ratio biologique/conventionnel du coût du travail dans la production de sorgho. La ligne bleue représente le seuil pour lequel les coûts de main-d'œuvre dans les systèmes biologiques et conventionnels sont identiques. Chaque point indique la médiane de la distribution élicitée.

Fig. 4. Median and credibility intervals (80\%) of organic to conventional ratio of labor cost in sorghum production.

\section{Une agriculture biologique moins productive mais plus rémunératrice sur les marchés locaux}

En moyenne, sur toutes les cultures et tous les pays, la médiane du ratio rendement biologique/rendement conventionnel est égale à 0,59 . En d'autres termes, la perte de rendement médiane liée à l'agriculture biologique est en moyenne de $41 \%$ par rapport à l'agriculture conventionnelle, d'après les 11 experts interrogés sur cette quantité. On observe que tous les intervalles de crédibilité incluent des valeurs strictement inférieures à la valeur 1 , à l'exception des experts 1 et 15 dont les intervalles coupent la valeur 1 (Fig. 3). Ces derniers considèrent donc possible d'avoir des rendements similaires avec les deux systèmes, voire un gain de rendement dans le système biologique. On constate des niveaux d'incertitude différents d'un expert à l'autre. Certains experts sont peu incertains, car leurs intervalles de crédibilité sont très petits, comme les experts 4 et 9 , et d'autres sont très incertains et fournissent de grands intervalles de crédibilité comme les experts 1 et 17 (Fig. 3).

Deux experts ont été interrogés sur la quantité de travail en nombre d'homme-jours dans la production du sorgho au Cameroun. Notre analyse révèle que les experts 2 et 3 considèrent que le coût du travail est plus élevé dans le système biologique que dans le système conventionnel (Fig. 4). Leurs intervalles de crédibilité couvrent des valeurs strictement supérieures à 1 . De plus, ils sont assez confiants dans leurs estimations car leurs intervalles de crédibilité sont relativement petits.

Deux experts ont été interrogés sur le coût des intrants dans la production d'oignon au Burkina Faso (Fig. 5). L'expert 17 prédit un coût plus faible dans le système biologique. Son intervalle de crédibilité couvre des valeurs strictement inférieures à 1 , et est très étroit. L'expert 15 est au contraire très incertain par rapport à l'expert 17. Son intervalle de crédibilité est très large et coupe la valeur 1 . Il considère donc possible que le coût des intrants dans le système biologique de l'oignon puisse être soit inférieur, soit supérieur à celui du système conventionnel. Néanmoins, la position de la médiane sur son intervalle de crédibilité (supérieure à 1 ) révèle qu'il accorde plus de $50 \%$ de chances au coût des intrants dans le système biologique d'atteindre des valeurs supérieures au système conventionnel. D'après l'expert 15 , le coût des 


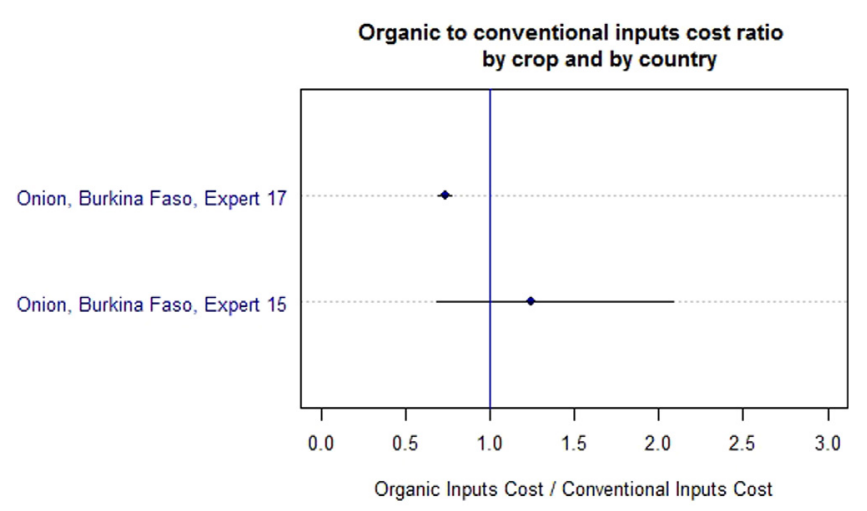

Fig. 5. Médiane et intervalles de crédibilité $(80 \%)$ du ratio biologique/conventionnel du coût des intrants dans la production d'oignon. La ligne bleue représente le seuil pour lequel les coûts des intrants dans le système biologique et conventionnel sont identiques. Chaque point indique la médiane de la distribution élicitée.

Fig. 5. Median and credibility intervals (80\%) of organic to conventional ratio of input cost in onion production.

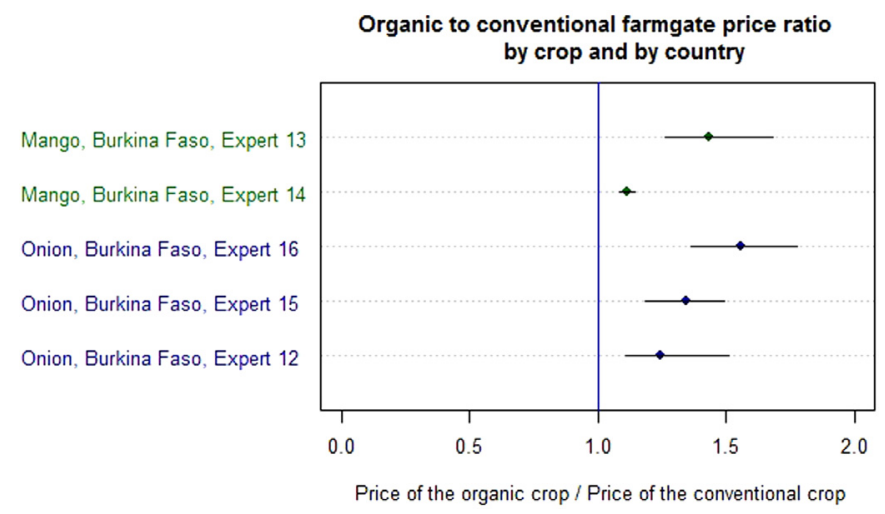

Fig. 6. Médiane et intervalles de crédibilité (80\%) du ratio biologique/conventionnel des prix bord-champ des produits agricoles. La ligne bleue représente le seuil pour lequel les prix des produits biologiques et conventionnels sont identiques. Chaque point indique la médiane de la distribution élicitée.

Fig. 6. Median and credibility intervals (80\%) of organic to conventional ratio of farmgate prices.

intrants est plus élevé dans les systèmes biologiques car les producteurs doivent souvent dépenser plus pour contrôler les ravageurs.

D'après les cinq experts interrogés, le ratio biologique/ conventionnel médian pour l'indicateur prix à la sortie de la ferme est de 1,34. Les intervalles de crédibilité des experts sont parfois grands, mais incluent toujours des valeurs strictement supérieures à 1 (Fig. 6). Les experts estiment que le prix des produits a plus de $80 \%$ de chance d'être plus élevé dans le système biologique que dans le système conventionnel. En revanche, selon l'expert, la différence peut être faible (expert 14) ou très élevée (expert 16).

D'après les huit enquêtes menées sur la durée de conservation des récoltes, nous avons obtenu un ratio biologique/conventionnel médian de 1,53 sur l'ensemble des

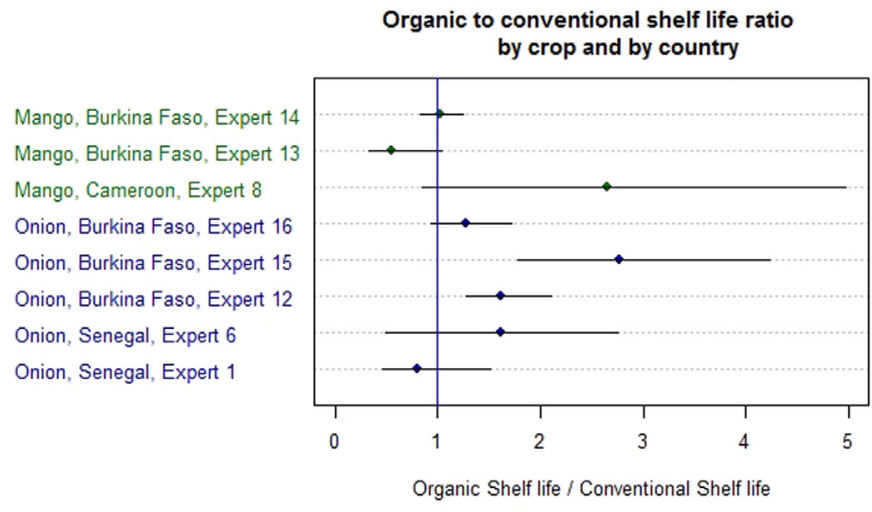

Fig. 7. Médiane et intervalles de crédibilité (80\%) du ratio biologique/conventionnel de la durée de conservation de produits frais. La ligne bleue représente le seuil pour lequel les durées de conservation des produits biologiques et conventionnels sont identiques. Chaque point indique la médiane de la distribution élicitée.

Fig. 7. Median and credibility intervals (80\%) of organic to conventional ratio of shelf life.

situations considérées. Néanmoins, les résultats sont variables et parfois contradictoires entre experts (Fig. 7). Seuls deux intervalles de probabilités sont clairement supérieurs à 1. Ils concernent les experts 12 et 15. Tous les autres intervalles incluent la valeur 1 .

Enfin, nous n'avons pas obtenu de résultats probants pour les indicateurs SDAM, fréquence de consommation d'aliments riches en vitamine A et fer, durée de conservation et contamination alimentaire par les mycotoxines. Les informations sur l'impact de l'agriculture biologique sur l'utilisation alimentaire n'ont pas pu être exploitées.

\section{Comparaison des résultats avec d'autres méthodes et nouvelles connaissances générées}

Les résultats obtenus sur les rendements, les prix des produits et les coûts des intrants permettent de tirer des conclusions sur les performances relatives de l'agriculture biologique par rapport à l'agriculture conventionnelle. Les résultats médians indiquent que les rendements biologiques sont estimés inférieurs d'environ $41 \%$ aux rendements conventionnels. Une méta-analyse basée sur des mesures expérimentales de rendement (Seufert et al., 2012) a montré que dans les pays en développement, les rendements biologiques sont en moyenne inférieurs de $43 \%$ aux conventionnels. Ainsi, notre étude donne des résultats comparables à ceux issus de données expérimentales.

Dans la littérature, on observe des prix supérieurs pour les produits biologiques certifiés, que ce soit dans les pays du Sud ou ceux du Nord (Crowder et Reganold, 2015). Dans notre étude, les experts n'ont pas seulement estimé des prix plus élevés pour les produits biologiques exportés, mais aussi pour ceux vendus sur le marché national (Fig. 6). Le marché local biologique peut offrir aussi bien des opportunités de profit aux commerçants que les marchés d'exportations (Tankam, 2017). 
Par exemple, au Burkina Faso, il existe un cadre institutionnel pour le secteur biologique, reconnu par l'IFOAM. Le CNABio (Conseil national de l'agriculture biologique) est une association ayant mis en place un système de certification biologique national, adapté au contexte local. Les produits biologiques, certifiés et labellisés "Bio SPG», sont vendus aux consommateurs locaux à fort pouvoir d'achat.

Nos résultats sur les coûts du travail sont originaux, car, pour autant que nous le sachions, il n'existe pas de données sur le travail en nombre d'homme-jours dans les conditions considérées dans cet article. Nous avons constaté que le nombre d'homme-jours requis est en moyenne plus élevé dans l'agriculture biologique. De nouvelles recherches sur les conséquences économiques et sociales de l'adoption de l'agriculture biologique sont nécessaires pour évaluer sa contribution à l'accessibilité alimentaire. En effet, une intensification par le travail augmente les coûts de production, mais elle peut aussi se traduire par une création d'emplois pour la population rurale.

L'intuition suggère que le coût des intrants dans le système biologique est généralement plus faible que dans le système conventionnel car les pratiques biologiques éliminent l'utilisation d'intrants chimiques (Crowder et Reganold, 2015). Pourtant, nous avons trouvé un ratio moyen proche de 1 . La comparaison des coûts des intrants dans les systèmes biologiques et conventionnels dans les pays d'Afrique subsaharienne nécessite des travaux supplémentaires.

Les résultats obtenus sur les indicateurs SDAM, fréquence de consommation en aliments riches en vitamine A et en fer, et risque de contamination par les mycotoxines, ne sont pas suffisants pour pouvoir être exploités. En effet, les experts ayant élicité ces indicateurs n'avaient jamais travaillé sur les systèmes de culture. Les méta-analyses publiées à ce jour en nutrition sont basées sur l'étude de nutriments isolés (Lairon, 2010; S rednicka-Tober et al., 2016), alors que nous avons sélectionné des indicateurs agrégés. Cette limite à notre étude soulève la question du choix de l'indicateur nutritionnel le plus adapté. Nos résultats sur l'indicateur « risque de contamination des aliments par les mycotoxines» ne sont pas non plus concluants. Nous n'avions pas trouvé d'experts ayant le profil adéquat pour éliciter cet indicateur.

Dans certains cas, il pourrait être intéressant d'agréger les distributions de probabilités produites par différents experts pour une même quantité dans un contexte donné. Cela permettrait de présenter les résultats de manière plus synthétique. Cependant, une telle approche doit être maniée avec précaution car elle peut gommer des divergences importantes entre experts.

\section{Conclusion}

Cette étude montre que l'élicitation probabiliste peut répondre aux besoins de quantification des informations en situation de carence de données expérimentales, situation que l'on rencontre fréquemment en Afrique subsaharienne. Dans le cas présent, cette méthode a permis de construire des indicateurs quantitatifs, mettant en relation l'agriculture biologique et plusieurs piliers de la sécurité alimentaire. Par ailleurs, les informations ont pu être collectées rapidement (en deux mois) et à faible coût. Une approche expérimentale aurait nécessité des moyens humains et matériels beaucoup plus importants et aurait également pris beaucoup plus de temps (au moins une saison de culture). La principale limite de cette méthode concerne l'identification d'experts disposant de connaissances tangibles sur les quantités élicitées. Pour certaines quantités, comme le rendement ou le prix des produits, l'identification d'experts compétents a été assez facile. Mais pour d'autres, notamment sur la qualité nutritive des produits ou la teneur en mycotoxines, nous n'avons pu identifier qu'un petit nombre d'experts, donnant des résultats contradictoires.

L'un des avantages de l'élicitation probabiliste est la description du niveau d'incertitude. Celui-ci s'est avéré être parfois élevé, parfois faible selon les cultures, les quantités élicitées et les experts. Cependant, certains résultats sont assez robustes. Ainsi, les résultats élicités indiquent des baisses de rendement substantielles dans les systèmes d'agriculture biologique, cohérentes avec celles rapportées expérimentalement dans d'autres régions du monde. Nos résultats indiquent par contre des prix de vente plus élevés pour les produits biologiques. D'après les experts, le coût en main-d'œuvre est plus élevé en agriculture biologique qu'en agriculture conventionnelle. Les résultats obtenus pour les autres quantités sont moins robustes. Il semble nécessaire de réaliser de nouvelles élicitations avec un panel plus large d'experts spécialisés sur ces autres indicateurs.

Remerciements. Ce travail a été financé en partie par l'institut Carnot Plant2Pro (projet L-i-cite), ainsi que par le projet ABASS (méta-programme INRA-CIRAD GloFoods). Les auteurs remercient les experts en France, au Burkina Faso, au Cameroun et au Sénégal, qui ont participé aux séances d'élicitation.

\section{Références}

Albert I, Ancelet S, David O, Denis JB, Makowski D, Parent E et al. 2015. Initiation à la statistique bayésienne. Bases théoriques et applications en alimentation, environnement, épidémiologie et génétique. Paris : Editions Ellipses. https://hal.archives-ouvertes.fr/ hal-01194144/

Berezow A. 2017. Organic farms use more land and don't decrease carbon footprint. New York, USA : American Council on Science and Health. https://www.acsh.org/news/2017/05/26/organic-farmsuse-more-land-and-dont-decrease-carbon-footprint-11338.

Clemen RT, Reilly T. 1999. Correlations and copulas for decision and risk analysis. Manag. Sci. 45: 208-224.

Cohen S, Chechile RA. 1997. Probability distributions, assessment, and instructional software: Lessons learned from an evaluation of curricular software. In: Gal I, Garfield JB, eds. The assessment challenge in statistics education. Amsterdam: IOS Press, pp. 253-267.

Crowder DW, Reganold JP. 2015. Financial competitiveness of organic agriculture on a global scale. Proc. Natl. Acad. Sci. 112: 7611-7616.

de Bon H, Huat J, Parrot L, Sinzogan A, Martin T, Malézieux E et al. 2014. Pesticide risks from fruit and vegetable pest management by small farmers in sub-Saharan Africa. A review. Agron. Sustain. Dev. 34: 723-736.

Dury S, Vall E, Imbernon J. 2017. Production agricole et sécurité alimentaire en Afrique de l'Ouest. Cahiers Agricultures 26: 61001. DOI: $10.1051 /$ cagri/2017047S. 
EFSA. 2014. Guidance on expert knowledge elicitation in food and feed safety risk assessment. EFSA J. 12: 3734.

Garthwaite PH, Kadane JB, O'Hagan A. 2005. Statistical methods for eliciting probability distributions. J. Am. Stat. Assoc. 100: 680-701.

Lairon D. 2010. Nutritional quality and safety of organic food. A review. Agron. Sustain. Dev. 30: 33.

Lesur-Dumoulin C, Malézieux E, Ben-Ari T, Langlais C, Makowski D. 2017. Lower average yields but similar yield variability in organic versus conventional horticulture. A meta-analysis. Agron. Sustain. Dev. 37: 45.

Makowski D, Albert I, Bonvallot N, Boudia S, Brochot C, Bruyere O et al. 2016. Prise en compte de l'incertitude en évaluation des risques: revue de la littérature et recommandations pour l'Anses. Rapport d'expertise collective. Maisons-Alfort: ANSES, 90 p. https://hal.archives-ouvertes.fr/hal-01617680/.

Morris DE, Oakley JE, Crowe JA. 2014. A web-based tool for eliciting probability distributions from experts. Environ. Model. Softw. 52: 1-4.

Oakley J. 2010. Eliciting univariate probability distributions. Rethinking Risk Measurement and Reporting 1. http://citeseerx.ist.psu.edu/ viewdoc/download?doi=10.1.1.461.8540\&rep=rep1\&type=pdf.

O'Hagan A. 2012. Probabilistic uncertainty specification: Overview, elaboration techniques and their application to a mechanistic model of carbon flux. Environ. Model. Softw. 36: 35-48.
S rednicka-Tober D, Barański M, Seal CJ, Sanderson R, Benbrook C, Steinshamn H et al. 2016. Higher PUFA and n-3 PUFA, conjugated linoleic acid, $\alpha$-tocopherol and iron, but lower iodine and selenium concentrations in organic milk: a systematic literature review and meta- and redundancy analyses. Br. J. Nutr. 115: 1043-60. DOI: $10.1017 /$ S0007114516000349.

Seufert V, Ramankutty N. 2017. Many shades of gray - The contextdependent performance of organic agriculture. Sci. Adv. 3.

Seufert V, Ramankutty N, Foley JA. 2012. Comparing the yields of organic and conventional agriculture. Nature 485: 229-232.

Tankam C. 2017. L'organisation des marchés de producteurs de fruits et légumes biologiques à Nairobi, Kenya. Cahiers Agricultures 26: 35006.

Temple L, Touzard JM, Kwa M, Boyer J, Requier Desjardins D. 2015. Comparaison des trajectoires d'innovation pour la sécurisation alimentaire des pays du Sud. Biotechnol. Agron. Soc. Environ. 19 (1): 53-61.

Touzard JM, Temple L. 2012. Sécurisation alimentaire et innovations dans l'agriculture et l'agroalimentaire : vers un nouvel agenda de recherche? Une revue de la littérature. Cahiers Agricultures 21: 293-301. DOI: 10.1684/agr.2012.0b/.

Yan J. 2007. Enjoy the joy of copulas: with a package copula. J. Stat. Softw. 21: 1-21.

Citation de l'article : Andriamampianina L, Temple L, de Bon H, Malézieux E, Makowski D. 2018. Évaluation pluri-critères de l'agriculture biologique en Afrique subsaharienne par élicitation probabiliste des connaissances d'experts. Cah. Agric. $27: 45002$. 